\title{
Technè
}

La science au service de l'histoire de l'art et de la préservation des biens culturels

41 | 2015

Arts textiles antiques et modernes. Approche scientifique et restauration

\section{Les détrempes sur toile du musée des Beaux-Arts de Reims : un corpus exceptionnel, une restauration d'envergure, un protocole de nettoyage inédit}

Distemper paintings on canvas at the Musée des Beaux-Arts, Reims : an

exceptional corpus, large-scale restoration and unprecedented cleaning protocol

Lydiane Chomienne et Claire Beugnot

\section{OpenEdition}

Journals

Édition électronique

URL : http://journals.openedition.org/techne/4796

DOI : 10.4000/techne.4796

ISSN : 2534-5168

Éditeur

C2RMF

Édition imprimée

Date de publication : 25 novembre 2015

Pagination : $94-100$

ISBN : 978-2-7118-6248-1

ISSN : 1254-7867

Référence électronique

Lydiane Chomienne et Claire Beugnot, "Les détrempes sur toile du musée des Beaux-Arts de Reims un corpus exceptionnel, une restauration d'envergure, un protocole de nettoyage inédit », Technè [En ligne], 41 | 2015, mis en ligne le 08 août 2020, consulté le 11 mars 2021. URL : http:// journals.openedition.org/techne/4796; DOI : https://doi.org/10.4000/techne.4796

La revue Technè. La science au service de l'histoire de l'art et de la préservation des biens culturels est mise à disposition selon les termes de la Licence Creative Commons Attribution - Pas d'Utilisation Commerciale - Pas de Modification 4.0 International. 


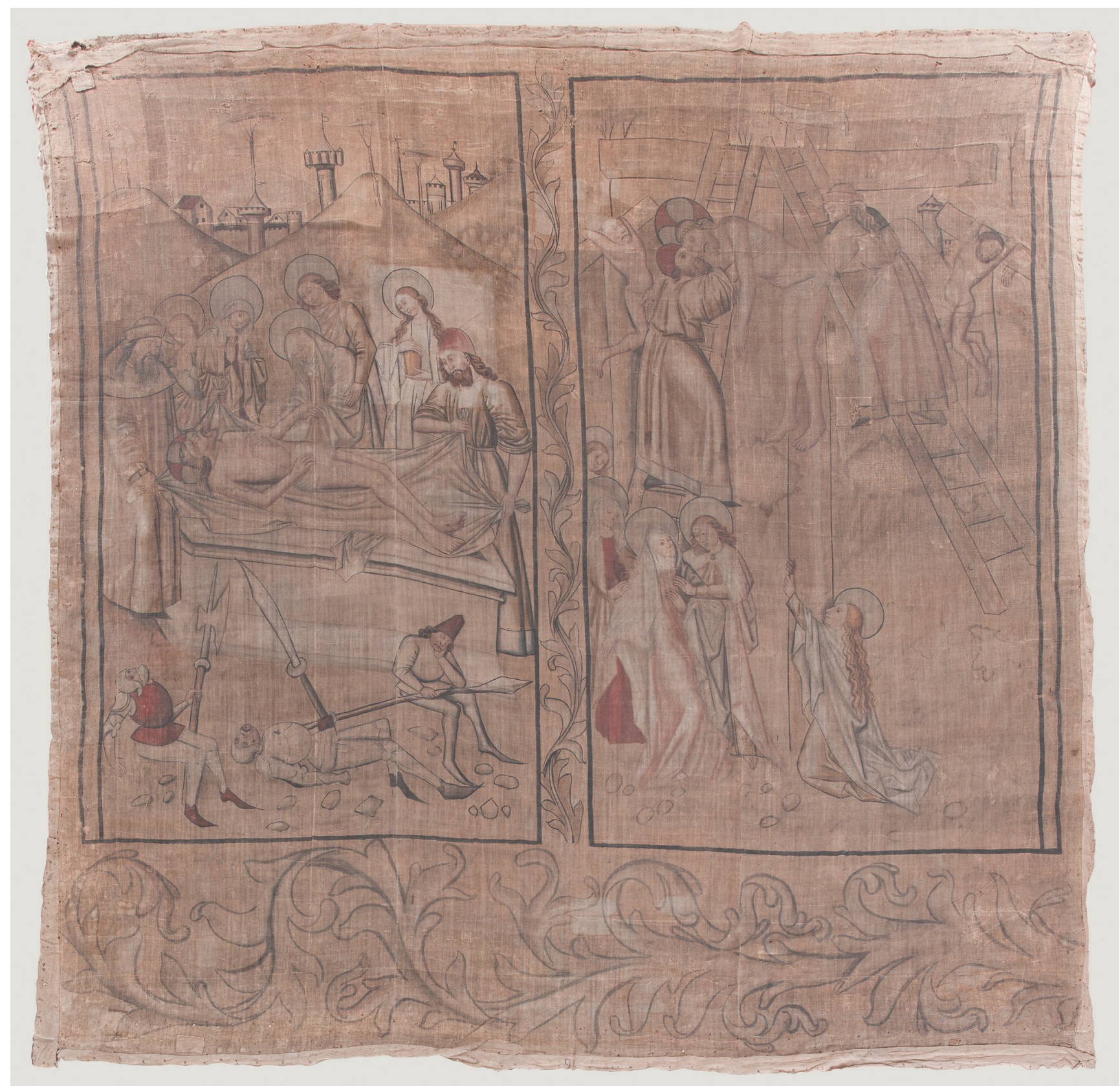

Fig. 1. La Mise au tombeau-Descente de croix avant restauration. ๑ C2RMF/Pierre-Yves Duval. 
Lydiane Chomienne

Claire Beugnot

\section{Les détrempes sur toile du musée des Beaux-Arts de Reims : un corpus exceptionnel, une restauration d'envergure, un protocole de nettoyage inédit}

Distemper paintings on canvas at the Musée des Beaux-Arts, Reims: an exceptional corpus, large-scale restoration and unprecedented cleaning protocol

\begin{abstract}
Résumé. Le musée des Beaux-Arts de Reims conserve un corpus de vingt-cinq toiles peintes de très grand format des $X V^{e}-X V I^{e}$ siècles, représentant des Saints et des Apôtres, des scènes du Nouveau Testament, ou de la Passion du Christ.

Ces détrempes sur toile libre, caractérisées par une couche picturale extrêmement fragile appliquée sur un support textile, ont subi une histoire matérielle mouvementée : reprisées, consolidées par rentoilage ou doublage, et profondément encrassées, leur état nécessitait une restauration globale de la collection. Le traitement, validé sur une première toile (la Mise au tombeau-Descente de croix) a requis la mise au point d'une méthode de nettoyage inédite à l'aide de membranes perméables. Le retrait des anciens doublages et la consolidation par couture sur support teint avec des teintures naturelles ont permis de considérer à nouveau cette auvre comme un textile peint et non plus comme une peinture traditionnelle.

Mots-clés. Toiles peintes, détrempes, membranes perméables, teintures naturelles.
\end{abstract}

\begin{abstract}
The Musée des Beaux-Arts, Reims, has a collection of 25 very large-scale painted canvases dating from the 15th-16th centuries, representing saints and the Apostles, scenes from the New Testament and the Passion of Christ. These distemper paintings on unframed canvas, all featuring an extremely fragile layer of paint on a textile support, had had an eventful material history: amendments had been made; they had been remounted or lined in order to be strengthened; they were extremely dirty. Due to their poor condition, a global restoration of the collection was required. The treatment, tested and approved on the first canvas (The Entombment-Descent from the Cross), involved developing an unprecedented cleaning process using permeable membranes. Removing old linings and consolidating the canvas by sewing it onto a support dyed with natural dyes enabled us to consider this work as a painted textile again, and no longer as a traditional painting.
\end{abstract}

Keywords. Painted canvases, distemper, permeable membranes, natural dyes.
La Mise au tombeau-Descente de croix ( $\mathrm{n}^{\circ}$ inv. D 876.1.13) (fig. 1) fait partie d'un ensemble exceptionnel conservé au musée des Beaux-Arts de Reims, constitué de vingt-cinq détrempes sur toile exécutées entre 1460 et le début du XVI ${ }^{\mathrm{e}}$ siècle pour orner l'Hôtel-Dieu de la cité rémoise. Ce corpus est unique en Europe car il illustre à grande échelle, en termes de nombre et de format des œuvres, la technique particulière de détrempe peinte sur toile libre. Or, cette technique, très répandue dans l'Europe du Xve siècle, constituait un patrimoine immense mais aujourd'hui en grande partie disparu en raison de l'extrême fragilité de ces œuvres. Les toiles de Reims constituent donc un témoin technologique précieux.

Ces objets singuliers se situent à la frontière entre peintures traditionnelles sur toile et œuvres textiles. En raison de leur grande fragilité liée à la technique d'exécution, elles ont soulevé de nombreux problèmes de conservation, et les multiples tentatives de restaurations effectuées ces dernières décennies se sont avérées inadéquates et destructrices ${ }^{1}$.

La restauration de la Mise au tombeau-Descente de croix a une dimension particulière et expérimentale. Elle est le résultat de plusieurs années d'investigations que nous avons menées sur ce type d'œuvres, et illustre une méthodologie inédite, ainsi que des procédés techniques innovants.

\section{Technique et historique des détrempes sur toile}

Il s'agit de peintures exécutées sur une toile souple et non préparée, tel un textile domestique. Ces toiles sont conçues pour être laissées libres, flottantes ou suspendues, et non pas tendues sur un châssis.

Le choix de la technique picturale participe également à une volonté d'exécution rapide et à une intention esthétique particulière. La couche colorée est simplement détrempée à l'eau, la facture est fluide et une partie importante de la toile est laissée en réserve. Les liants les plus répandus sont les colles animales ou les gommes végétales ${ }^{2}$. Ces peintures sont laissées telles quelles, sans vernis, et présentent donc un aspect poreux et mat caractéristique. Cette technique permet, à l'inverse des panneaux de bois utilisés à cette époque, une

Lydiane Chomienne, restauratrice de peintures (lydianechomienne@ hotmail.com). Claire Beugnot, restauratrice de textiles (claire.beugnot@ orange.fr). 
mise en œuvre peu coûteuse pour des œuvres légères et facilement transportables, même dans des formats importants. Leurs destinations sont donc multiples. Elles remplacent aisément tapisseries et peintures murales ou sont parfois à destination portative comme les bannières de procession. D'autres usages, qu'ils soient liturgiques ou éphémères (théâtre, processions royales), ont également été relevés ${ }^{3}$.

Cette production s'avère donc multiple et massive dans l'Europe du Xve siècle. Elle dépasse largement les exemples plus connus des prestigieux tüchlein nordiques ou des peintures de Mantegna ${ }^{4}$.

Quant aux toiles de Reims, leur fonction précise soulève encore de nombreux questionnements, mais il semblerait toutefois qu'elles aient été commandées pour elles-mêmes, plutôt que comme éléments préparatoires ${ }^{5}$.

\section{Histoire matérielle du corpus de Reims}

Ces détrempes « rémoises » sont par nature très fragiles : le support n'est pas tendu ni maintenu ; le liant aqueux est très soluble et la couche picturale est particulièrement sensible à l'abrasion, d'autant plus qu'il n'y a pas de vernis protecteur. De par cette fragilité, mais aussi de par leur singularité technique mal comprise au cours du temps, elles ont subi une histoire matérielle complexe et mouvementée. D'après l'étude de Monique Favre-Communal ${ }^{6}$, il semblerait que ces toiles n'aient pas été conçues pour être éphémères. Un nouveau travail de recherche historique s'imposerait pour éclaircir leur fonction.

L'entretien des toiles figure de manière éparse et ponctuelle dans les archives du musée. Puis les choses s'intensifient ces dernières décennies car il semblerait que leur état soit devenu une préoccupation permanente. Des années 1960 aux années 1990, plusieurs campagnes de restauration se succèdent, aboutissant à des impasses techniques ${ }^{7}$. Les œuvres sont traitées comme des peintures traditionnelles sur toile, et subissent des interventions structurelles fondamentales telles que des doublages et des rentoilages, provoquant ainsi la perte de leur identité particulière. Par ailleurs, les tentatives de nettoyage par bains de trempage, ou sur table aspirante, provoquent un lessivage irréversible de la matière picturale.

Il était donc impératif d'innover, tant dans l'approche des œuvres que dans les protocoles d'intervention. C'est ce que notre équipe pluridisciplinaire a réalisé lors de l'étude préalable $^{8}$ de 2009 , puis mis en pratique lors de la restauration de la Mise au tombeau ${ }^{9}$.

Cette méthodologie, fondée sur la connaissance approfondie ${ }^{10}$ des caractéristiques techniques spécifiques aux détrempes sur toile, s'est constituée autour de deux axes : le traitement de l'œuvre comme un textile peint, et non plus comme une peinture traditionnelle, et la mise au point expérimentale d'un procédé de nettoyage.

\section{Restauration}

\section{Constat d'état}

La Mise au tombeau est peinte sur une toile de chanvre ${ }^{11}$ constituée de trois lés cousus verticalement. Elle mesure $357 \mathrm{~cm} \mathrm{x}$ $348 \mathrm{~cm}$.

Le tissu de support de la détrempe est une toile écrue moyenne, assez régulière et de tissage serré (réduction chaîne : 13 fils/cm, torsion Z ; trame : 8-9 coups/cm, torsion Z). Elle est libre et n'a pas fait l'objet de traitements fondamentaux.

La palette est réduite et principalement constituée de couleurs terre et de noir. Apparaissent également des plages colorées rouges, et plus ponctuellement, des rehauts de blanc et des tracés verts constitués de vert au cuivre et de jaune d'arsenic ${ }^{12}$. Une partie importante de la toile est gardée en réserve (fig. 2).

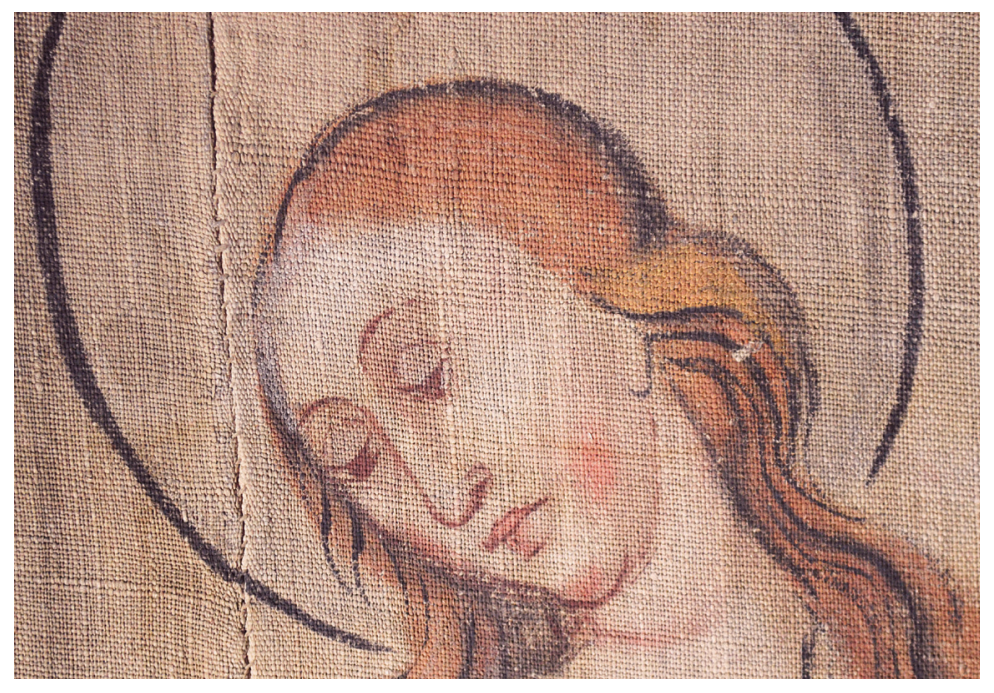

Fig. 2. La technique picturale de détrempe sur toile mise en valeur après le nettoyage. (C) Lydiane Chomienne.

Les motifs sont cernés de noir et les volumes sont définis par des traits, plus ou moins ombrés, ainsi que par des dégradés en lavis. La mise en couleur est exécutée de manière très fluide et en couche unique. Quelques superpositions sont toutefois repérables au niveau des rehauts lumineux ou de certaines zones ombrées. Les couches colorées sont extrêmement fines, elles ne forment pas de film de surface et ont simplement coloré le textile. L'observation sous faible grossissement permet de visualiser les grains de pigments agglomérés et accrochés sur les fibres.

Il n'y a aucune trace d'encollage visible, mais le fantôme de la composition n'apparaît pas au revers de l'œuvre, signe d'une imprégnation efficace des fibres. 
Le liant n'a pas été identifié par des analyses, mais il s'agirait d'un liant aqueux (gomme ou colle animale), ce qui serait en accord avec la littérature ${ }^{13}$.

L'état de conservation de l'œuvre est révélateur de la fragilité de la technique face aux agents dégradants extérieurs tels que les manipulations, les montages successifs, la lumière et l'humidité.

La toile est marquée par des lignes de pliage ancien.

La couleur de la toile de fond s'est modifiée et n'est plus uniforme. Elle est généralement jaunie, ce qui peut être un effet d'exposition à la lumière lors de sa présentation, et noircie par l'empoussièrement. Localement, elle est plus claire, car tachée et marquée par des auréoles caractéristiques d'humidité (grandes taches plus claires avec un front plus foncé irrégulier) ou de l'action de micro-organismes (petites taches rondes et claires).

Sur la toile, des déchirures et des lacunes apparaissent, notamment en périphérie, attestant de montages et accrochages successifs cloués, comme le montrent les lignes de petits trous portant des traces de rouille, régulièrement disposés.

On note également des bandes de renfort le long des bords ou dans les angles.

Des lacunes sont dispersées sur l'ensemble de la toile. Elles ont été consolidées anciennement par pose de pièces locales ou par des reprises. Les pièces qui proviennent de tissu de remploi sont des fragments de toile de lin ou de chanvre, parfois de toile peinte, teinte ou imprimée. Une des pièces est brodée au point de croix. On note quelques inscriptions à l'encre au dos de la toile peinte.

Ces pièces ont fait écran à l'empoussièrement et la couleur de la toile d'origine, en dessous, est restée plus claire.

Les reprises souvent effectuées avec un fil de lin grossier sont très débordantes et visibles : elles peuvent être particulièrement disgracieuses car couvrant des zones figuratives, par exemple le visage de la Vierge.

Pour la couche picturale, de nombreuses usures, abrasions et épidermages apparaissent. Ceci témoigne de la sensibilité de la peinture aux frottements. Par ailleurs, la lisibilité est extrêmement perturbée par un empoussièrement et un encrassement très prononcés, ainsi que par la présence d'auréoles, de taches et de salissures de surface.

\section{Dépose des reprises anciennes}

Après concertation avec le comité de suivi ${ }^{14}$, la première intervention a consisté à débarrasser la toile peinte de tous les matériaux qui ont été rapportés, afin de l'assainir. Les pièces ont été documentées et conservées dans le dossier d'œuvre. Le retrait s'est fait à l'aide de ciseaux à mouches et de pinces. Cette opération s'est révélée assez délicate, car le fil dégradé ne glissait pas et il a fallu le couper point par point (fig. 3 et 4 ).

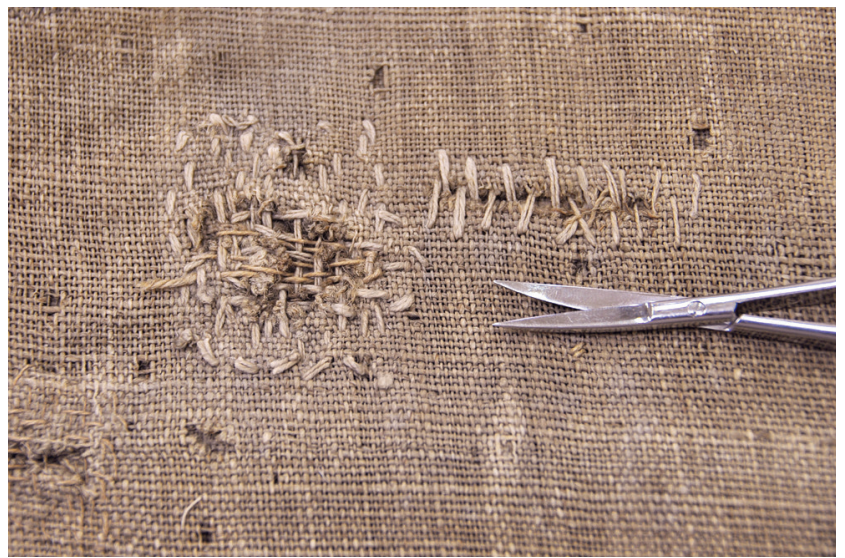

Fig. 3. Les reprises très couvrantes ôtées au ciseau à mouches et à la pince. (C) Claire Beugnot.

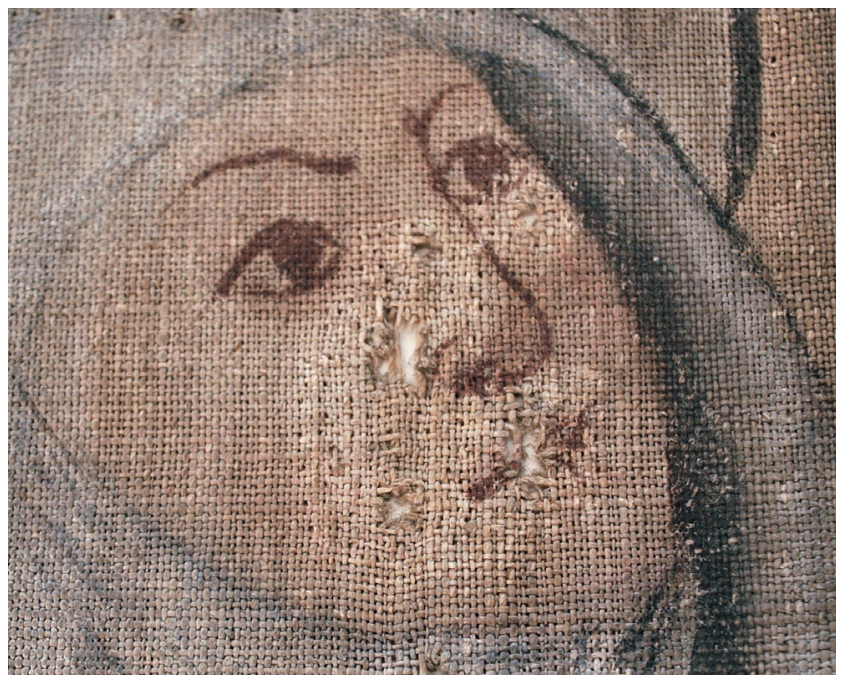

Fig. 4. Détail de la Vierge après dépose des reprises anciennes et avant nettoyage et consolidation. (C) Lydiane Chomienne.

\section{Nettoyage}

Après un dépoussiérage délicat et minutieux, nécessitant de ne pas faire glisser l'embout de l'aspirateur sur les zones peintes afin de ne pas les abraser, s'est déroulé le travail de nettoyage aqueux à proprement parler.

La difficulté intrinsèque au décrassage de cette ouvre réside dans le fait que le recours à l'eau est indispensable pour solubiliser et retirer la crasse accumulée dans la matière, mais que l'eau est justement l'agent dégradant de la peinture. Par ailleurs, la fragilité mécanique des couches colorées exclut toute action de frottement. Enfin, la porosité extrême empêche le recours aux méthodes laissant des résidus. 


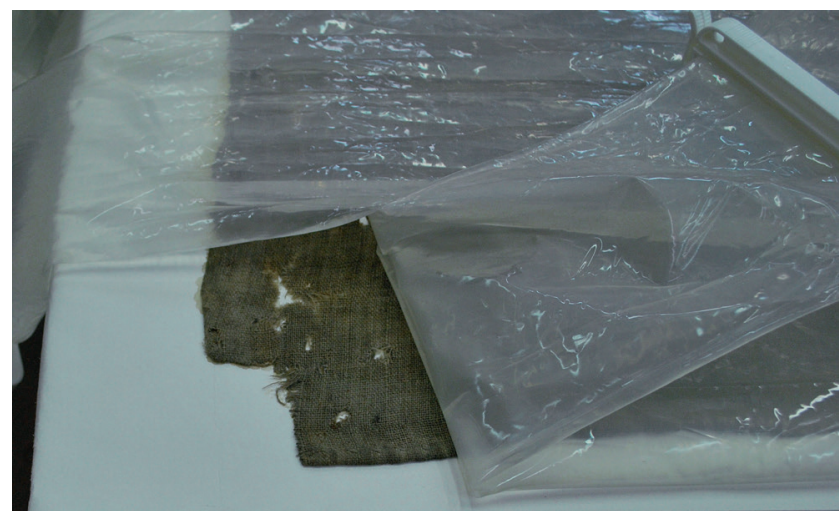

Fig. 5. Les membranes de nettoyage appliquées à la surface de l'œuvre. (C) Lydiane Chomienne.

Le champ d'action est donc considérablement réduit. Et les systèmes généralement employés dans ce type de problématique, tels que les compresses diverses et variées avec pulpe de cellulose, papiers absorbants, cataplasmes d'argile, gels à haute densité, table aspirante, ne permettent pas de répondre à ce cahier des charges excessivement contraignant. Le contrôle de l'eau n'est pas assuré, risquant d'entraîner la formation d'auréoles et la solubilisation partielle de la matière colorée ${ }^{15}$.

Une recherche expérimentale a donc été menée afin de trouver un système statique, sans action mécanique, qui permette un apport d'eau contrôlé au sein de la matière poreuse, puis son extraction, ainsi que celle de la crasse solubilisée.

Nous avons retenu des membranes perméables en cellulose régénérée, dont la porosité de l'ordre moléculaire permet des phénomènes physiques d'échange d'ions entre deux milieux. Ces membranes en forme de «boyaux synthétiques ${ }^{16}$ sont largement utilisées dans l'agro-alimentaire. Elles permettent d'enfermer l'agent actif (eau déminéralisée), puis lorsqu'elles sont mises en contact avec l'œuvre, les mécanismes d'échange se mettent alors en place de part et d'autre. L'eau est libérée dans le substrat original poreux, puis remonte dans le boudin, chargée de crasse et des produits de dégradation ionisés contenus dans le textile. Ce mécanisme se déploie selon une cinétique extrêmement lente ${ }^{17}$, pendant plusieurs heures, assurant une humidification progressive des matériaux originaux, préférable pour le textile, ainsi qu'un contrôle optimal de la captation de la crasse hydrosoluble. Le système est statique, et les forces physiques en présence de part et d'autre de la membrane sont telles qu'il n'y a pas de diffusion latérale : aucune auréole n'apparaît et la surface de nettoyage correspond strictement à la surface de contact avec le boyau (fig. 5).

Sur la Mise au tombeau, nous avons fait varier les paramètres de mises en ouvre (temps de pose, renouvellement des boudins, superposition avec d'autres membranes ou buvard), jusqu'à obtenir le protocole le plus satisfaisant possible. Le procédé s'est avéré reproductible, ce qui a permis d'obtenir sur ce grand format une surface nettoyée régulière et sans démarcation.

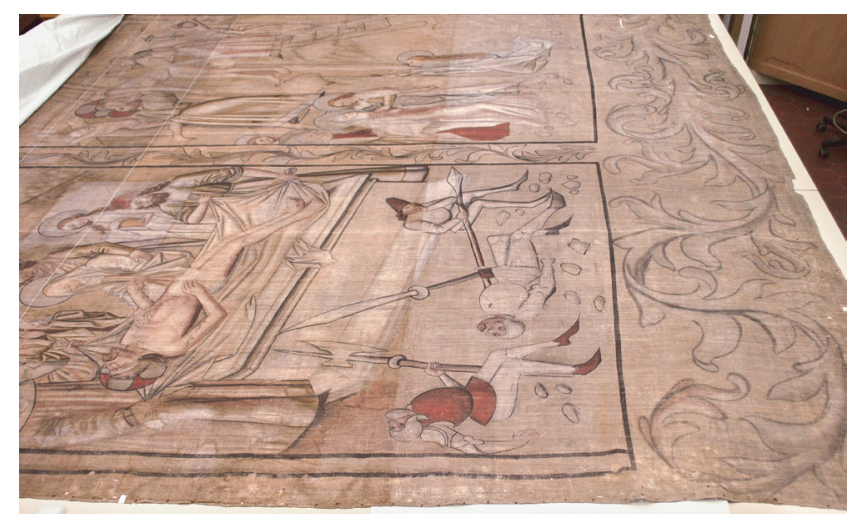

Fig. 6. L'œuvre en cours de nettoyage : à gauche avant dépoussiérage, au centre après dépoussiérage, à droite après nettoyage aqueux. (c) Lydiane Chomienne.

L'effet du dépoussiérage puis du nettoyage avec membranes est donc très satisfaisant. Les scènes de la Passion ont retrouvé leur luminosité, leurs contrastes, ainsi que leur chromatisme subtil (fig. 6).

Après concertation avec le comité scientifique, et dans l'optique d'un traitement minimaliste, il a été décidé de ne pas recourir à la réintégration picturale des lacunes et usures de la matière colorée $\mathrm{e}^{18}$.

\section{Consolidation textile}

La seconde phase du traitement concerne la consolidation de la toile peinte afin de permettre un accrochage à la verticale comme pour une tapisserie.

Les lacunes ponctuelles sont consolidées sur des pièces de support locales et les zones fragilisées maintenues par des points de restauration au fil de soie, points très discrets qui se perdent dans l'épaisseur de la toile d'origine (fig. 7). Pour les bords où les lacunes sont nombreuses, la consolidation se fait sur des bandes de la largeur ou de la hauteur totale.

Les pièces sont teintes à l'aide de teintures naturelles ${ }^{19}$, adaptées au support et à son ancienneté (toile de chanvre du $\mathrm{XV}^{\mathrm{e}}$ siècle) et au ton recherché (un gris beige assez clair).

La pose d'une doublure est ensuite effectuée afin de supporter et répartir le poids de l'ensemble de la toile peinte et d'en protéger l'arrière. Après préparation, la doublure est fixée par des lignes de points en quinconce avec une soie surfine. L'accrochage se fera au moyen d'une barre métallique glissée dans un manchon cousu.

La restauration de cette toile peinte a été menée par une équipe pluridisciplinaire en étroite concertation avec les conservateurs et le comité de suivi. Elle a été réalisée en respectant les fragilités de l'œuvre, mais également l'environnement : méthodes de nettoyage par membranes perméables sans solvants, teintures naturelles, consolidation par couture. Le résultat spectaculaire permet de retrouver une toile débarrassée des salissures et des éléments additionnels, avec présentation à la verticale dans une configuration plus proche de ce qu'elle est : une toile libre. En dehors des périodes de 


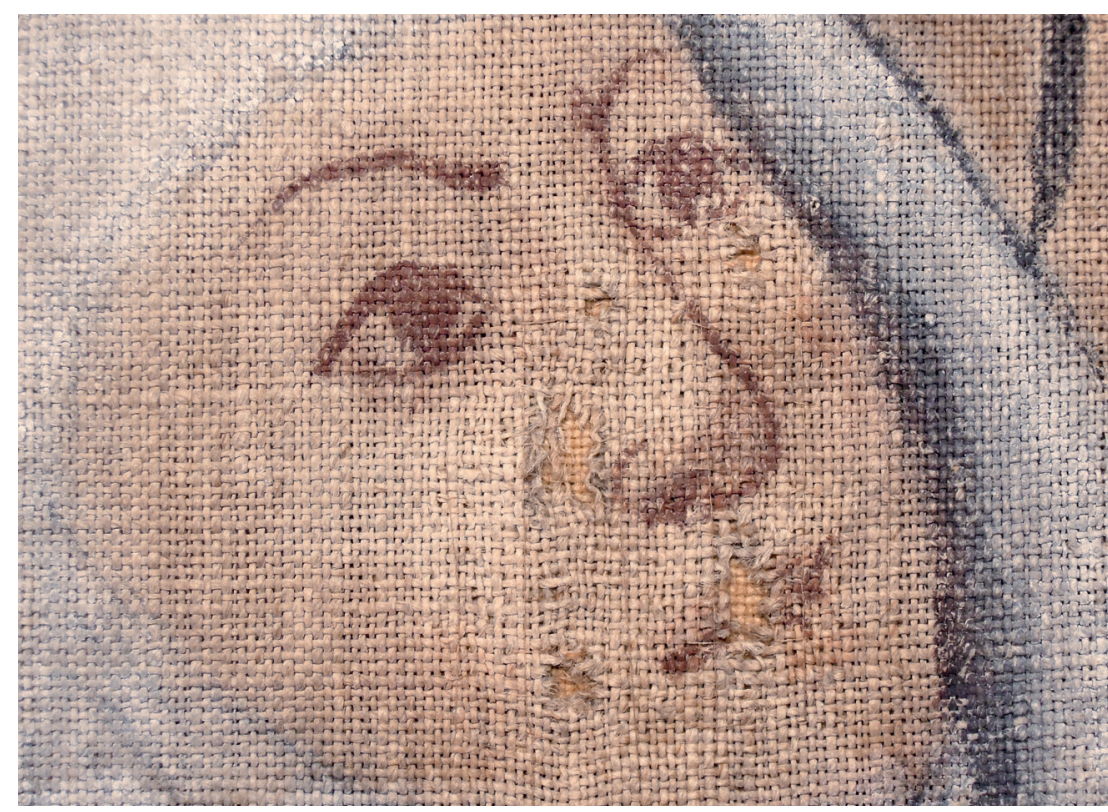

Fig. 7. Détail de la Vierge après restauration : les personnages ont retrouvé leur modelé et les points de restauration au fil de soie n'altèrent pas la vision des visages. @ L Lydiane Chomienne.

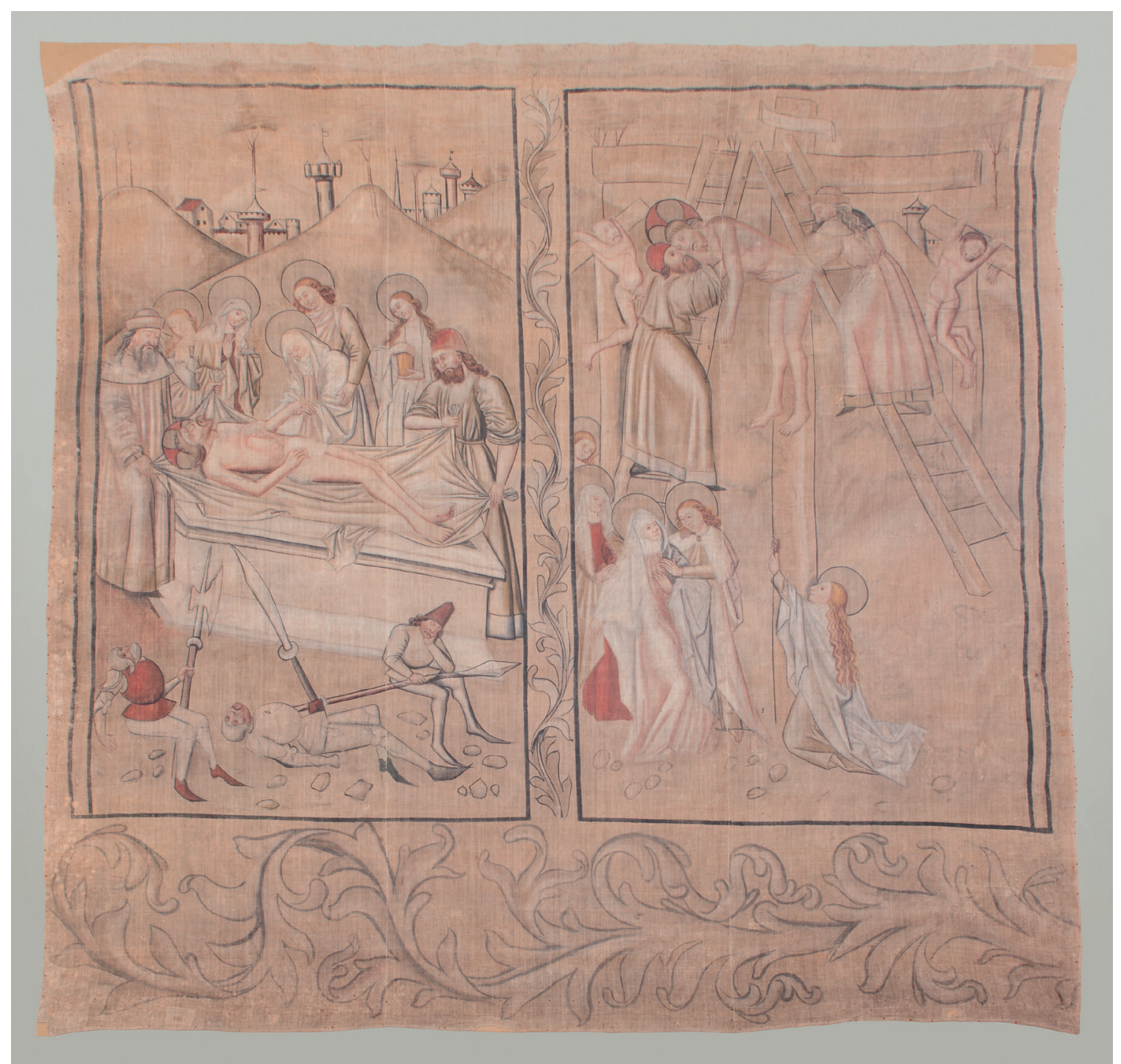

Fig. 8. L'œuvre après restauration. La toile est assainie, la surface nettoyée et la consolidation permet un accrochage comme un textile tout en maintenant les zones fragilisées. @ C C2RMF/Thomas Clot. 
présentation, la toile sera conservée sur un rouleau autoportant de large diamètre $(40 \mathrm{~cm})$ (fig. 8).

Ce traitement expérimental doit maintenant être appliqué à l'ensemble du corpus, afin de stopper les dégradations, restituer les qualités picturales et chromatiques des scènes et permettre la présentation des œuvres aux visiteurs. Chaque toile étant unique et présentant, en plus des dégradations vues ici, d'autres altérations (notamment des rentoilages et doublages synthétiques), nul doute que les restaurateurs auront à mobiliser toutes leurs ressources et leurs compétences pour répondre aux défis nouvellement posés, difficultés fécondes car elles permettent de développer de nouvelles approches de restauration.

\section{Notes}

1. Grebille, Lamotte, 1992.

2. Villers C., 2000.

3. Idem.

4. Rothe, 1995 ; Wolfthal, 1989.

5. Favre-Communal, 1992.

6. Favre-Communal, 1992.

7. Beugnot, Chomienne, Vibert, 2009 ; pour la mise au point des procédés de restauration, voir Rapport de Binenbaum sur la D.876.1.21, 1965, documentation du C2RMF/Rapport de Binenbaum sur la D.876.1.23, 1966, documentation du C2RMF. Le rapport de Grebille, Lamotte de 1992 évoque une remise en question des procédés utilisés jusqu'alors.

8. Le Groupement Chomienne ayant réalisé l'étude de mars à juin 2009 était constitué de Lydiane Chomienne (couche picturale), Christian Vibert, restaurateur de peintures (support) et Claire Beugnot (textiles).

9. Restauration effectuée en septembre 2011-août 2012 à Versailles, dans les ateliers de restauration du C2RMF. La restauration a été effectuée par Lydiane Chomienne, Christian Vibert, Claire Beugnot et Cécilia Aguirre, restauratrice de textiles. Rapport C2RMF 26037. 2007.

10. Chomienne, 2005 et Chomienne,

11. Sylvie Demailly, Laboratoire de Recherche des Monuments Historiques, rapport 880-A du 26.02.1992.

12. Sylvie Demailly, Laboratoire de Recherche des Monuments Historiques, rapport 880-A du 26.02.1992.

13. Villers, 2000.

14. Le comité de suivi réunissait les conservateurs responsables de l'œuvre: David Liot et Catherine Arnold que nous remercions pour leur confiance, ainsi que Claire Gérin-Pierre, conservateur à la filière Peinture au C2RMF, et Roberta Cortopassi, conservateur responsable de la filière Arts décoratifs au C2RMF.

15. Masschelein-Kleiner, 1978-1979.

16. Membranes de cellulose régénérée VISKASE HS.

17. Bouzid, 2002.

18. Après restauration des autres œuvres de la série et dans l'optique d'une éventuelle harmonisation du corpus, la question de la réintégration pourra à nouveau faire l'objet d'une réflexion.

19. Teintures naturelles réalisées par Cécilia Aguirre, à base de châtaignier et de garance avec mordançage à l'extrait de noix de galle et acétate d'aluminium.

Un nuançage avec fer, titane et alun permet d'ajuster la teinte et d'augmenter la solidité de la teinture.

\section{Références bibliographiques}

Chomienne L., « Démarche originale et techniques alternatives : restauration d'une détrempe sur toile italienne », Patrimoines, revue de l'Institut national du patrimoine, $\mathrm{n}^{\circ}$ 3, 2007, p. 107-113.

Masschelein-Kleiner L., « Examen et traitement d'une détrempe sur toile attribuée à Thierry Bouts :

la Crucifixion de Bruxelles ", Bulletin de l'IRPA, vol. XVII, Bruxelles, 1978/79, p. 5-21.

Rothe A., "Andrea Mantegna's Adoration of the Magi", Historical painting techniques materials and studio practice, Preprints of the Leyden Symposium, Los Angeles, Conservation Institute, 1995, p. 111-116.

Villers C., The Fabric Of Images: European Paintings On Textile Supports In The Fourteenth And Fifteenth Centuries, Proceedings of the international symposium held on 16 may 1998 at the Courtauld Institute, London Archetype publications, 2000, ouvrage collectif.

Wolfthal D., The Beginnings Of Netherlandish Canvas Painting: 1400-1530, Cambridge University Press, USA, 1989.

\section{Documents inédits}

Beugnot C., Chomienne L., Vibert C., Étude préalable à la restauration des toiles peintes appartenant aux collections $d u$ musée des Beaux-Arts de la ville de Reims, 2009, consultable sur demande à la documentation du C2RMF.

Bouzid T., Conserver ou retirer les interventions anciennes : étude de sept textiles islamiques médiévaux restaurés ou montés à la fin du $X I X^{e}$ siècle ou au début du XX $X^{e}$ siècle. Mise au point et expérimentation d'une méthode de traitement des textiles collés très fragilisés, à l'aide d'argiles contenues dans des membranes cellulosiques, mémoire de fin d'études INP, Saint-Denis, 2002.

Chomienne L., Étude et restauration d'un Christ au jardin des Oliviers (Paray-leMonial). Une énigmatique détrempe sur toile italienne du XVI ${ }^{e}$ siècle. Mise au point d'une technique de nettoyage d'une æuvre imprégnée et dénaturée, mémoire de fin d'études INP, Saint-Denis, 2005.

Favre-Communal M., Neuf toiles peintes conservées au musée de Reims - La Passion du Christ (fin du XV siècle), mémoire de muséologie de l'École du Louvre, Paris, 1992.

Grebille C., Lamotte A., Les toiles peintes du musée Saint-Denis de Reims, Série de la "Vengeance du Christ", mémoire de muséologie de l'École du Louvre, Paris, 1992.

Grebille C., Lamotte A., Les toiles peintes du musée Saint-Denis de Reims, Série de la "Vengeance du Christ ", Archives du C2RMF, Versailles, mars 1992. 